\title{
Workers' Occupational Hazards at Textile Factory in Damietta City
}

Ateya Megahed Ibrahim ${ }^{1}$ Prof. Effat Mohamed El-Karmalawy ${ }^{2}$ Ass. Prof.Mona AbdElsabour Hassan ${ }^{3}$ Dr. Fatma El-Emam Hafez ${ }^{4}$

${ }^{1}$ M.Sc. in Family and Community Health Nursing, Faculty of Nursing, Port Said

University. ${ }^{2}$ Professor of Community Health Nursing, Faculty of Nursing, Cairo

University. ${ }^{3}$ Associate Professor of Family and Community Health Nursing, Faculty of

Nursing, Port Said University. ${ }^{4}$ Lecturer of Family and Community Health Nursing,

Faculty of Nursing, Port Said University.

\begin{abstract}
Background: Occupational hazards that exist within the textile factory have a destructive and serious effect on the workers' health. The aim: of this study is to assess the workers' occupational health hazards at textile factory in Damietta City. Sample: the study is carried on 108 workers. Research design: A descriptive research design is utilized. Data collection tools: data are collected using two tools: workers' structured Interview Questionnaire, and workers' Observational Checklist. Results: revealed that two-fifths $(40.0 \%)$ of the study group were between the ages of 46-60 years. Less than three quarters $(71.3 \%)$ of the study group reported exposing to physical hazards, slightly more than two thirds $(69.4 \%)$ were exposed to chemical hazards, two thirds $(66.7 \%)$ were exposed to mechanical hazards and more than half $(59.3 \%)$ of the study group were exposed to psychological hazards. There was a statistically significant difference between levels of education of the study group and various types of occupational hazards, while there was no statistically significant difference between personal protective equipment and various types of occupational hazards. Conclusion: Most of the study group was exposed to various types of occupational health hazards at their workplaces which resulted in tremendous harmful effects on their health. Recommendations: The study recommended the need for stressing on the application of International Standard Occupational Health and Safety Assessment Series in field work to improve occupational health and safety performance of the workers and emphasizing on the importance and usefulness of personal protective equipment to be used in the right way. Key words: Occupational health hazards, Personal protective equipment, textile factory.
\end{abstract}




\section{INTRODUCTION}

Work is seen as important to one's life experience; most adults spend about fulfilling one third of their time at work (Rogers, 2015). Work when fulfilling, fairly compensated, healthy and safe can help to establish long and contented lives and strengthen families and residential district. Although some actors may never face more than minor adverse health effects from exposure at work, such as occasional eye strain resulting from poor office lighting, every industry grapples with serious risks. No work is completely risk-free and all health care professionals should deliver some basic knowledge about workforce populations, employment and related risks and methods to control risks and better health (Stanhope \& Lancaster, 2016).

The International Labor Organization (ILO), the World Health Organization (WHO) and United Nations (UN) have calculated that there are 270 million people suffer from occupational health hazards, and 2 million die as a result of these risks. Hazardous substance causes the destruction of an estimated 440,000 workers. In USA in one year, approximately 2 million workers were victims of work violence. In the UK, in the same year, 1.7 for working adults (357,000 workers) were the victims in one or more incidents of workplace risks. In summation, the estimated economic loss caused by work related hazards and diseases was equivalent to $4 \%$ of the world's gross national Product (Mousa, 2014 \& Hughes and Ferrett 2016).

Risks in the working environment can be divided into five primary classes. Chemical (dusts, liquids, fibers, fumes, mists and smoke), physical (noise, vibration, ionizing and non-ionizing radiation, vibration, and extreme of temperature), biological (bacteria, viruses, fungi, and protozoa), ergonomic and mechanical (lifting, repetitive action, military capabilities, and traps), psychosocial and organizational (work demand and conditions, work environment and organizational). These may produce an immediate or delayed response dictated largely by workers' inherent characteristics and the intensity and frequency exposure (Smedley and Sadhra 2013; Popov et al., 2016).

Personal protective equipment (PPE) belongs to a group of equipment that protects employees against dangers in the work. The decision to use such equipment must be preceded by all possible actions, both technical and organizational, aimed at 
eliminating or reducing the hazard to an admissible level . A frequent cause of accidents in the workplace is failure to use PPE by the workers or their wrong selection for the level of risk connected with the occurring hazards .workers reluctance to use PPE may result from the equipment not being well fitted to the needs of a user and additional conditions connected with work organization in a specific workplace (Salvendy, 2012).

The Textile production industry is one of the most precious and most technologically complex of all industries because of the variety of substrates, processes, machinery and components used and finishing steps undertaken (Hasanbeigi \& Price, 2012). Cotton is the main raw material followed by synthetic yarns (rayon and nylon). The production of cotton threads and materials involved in the following general processes: fiber preparation, Spinning (twisting/Texturing), weaving, pretreatment processing which includes (singeing, de-sizing, scouring, mercerizing, carbonizing, and bleaching), dyeing, printing and finishing of yarns and fabrics (Babu et al., 2007; Zabaniotou \& Andreou, 2010).

Textile industry contains many hazards and risks to workers because of exposure to noise, chemicals including dyes, solvents, optical brighteners, finishing agents and numerous types of natural and synthetic fiber dusts which affect their health, and airborne particulate which affect their health. Likewise, workers involved in manual treatment and working with machinery are at a risk of several health conditions. One of the most serious and sever diseases associated with the cloth industry and cotton dust are byssinosis which characterized by chest tightness, breaking problem, asthma and irritation in the Respiratory track (Lai and Christiania, 2014; Saramon, 2014; Singh, 2016).

The Occupational Health Nurse (OHN ) plays a major part in helping to protect and improve the health of working populations, preventing sickness and accidents, providing aid to the employee who is returning to work after an illness or injury, and ensuring a safe business or industrial surroundings. Passed on the expanding opportunities in this type of service nurses can market their expertise to employers. They can provide programs targeted at job-related safety, weight reduction, and dependence-free lifestyles as well as promote nutrition, physical exertion, smoking cessation, and family planning, empower employees to make informed health 
decisions while also overseeing the health dangers and costs connected with the employment relations between employees and the business (Guzys and Petrie, 2014; Hunt, 2009).

\section{Significance of the Study:}

Textile mills are a risky environment where workers are let out to different occupational hazards which may have tremendous harmful effects on their health, affects the morale and productivity of them.Moreover, it has been noted that actors are loath to comply with safety standards. Hence, it is rattling important to assess the workers' occupational hazards to assure dependable and sound working conditions, control risks, improve cost effectiveness, provide satisfaction, and protect the workers, supervisors and the organization assets. When identifying occupational hazards, it is an important facet of risk management. There had been no evidence of conducting previous studies on occupational health hazards to workers in a fabric factory in Damietta city; so this study was extended out to display the occupational health hazards and the preventive measures employed by the actors in their work in Damietta textile factory.

\section{AIM OF THE STUDY:}

The aim of the study is to assess workers' occupational health hazards at a textile factory in Damietta City.

\section{Research Objectives:}

1-To detect the occupational health hazards at the textile factory in Damietta City.

2-To identify the effects of the occupational health hazard exposure on workers' health at the textile factory in Damietta City.

3- To determine the safety measures that the workers use at the textile factory in Damietta City.

\section{SUBJECTS AND METHOD:}

\section{Study Design \& Setting}

A descriptive cross-sectional research design is used, carried out in the textile factory in Damietta city which consists of three large wards namely (pretreatment and Fiber preparation wards, Spinning and weaving wards and Dyeing and printing wards). 


\section{Total population}

Total number of workers in the textile factory were 120 workers

Study Sample: All workers in the textile factory (108 workers) were admitted in the study after exclusion of the pilot study (12 workers) from the entire sample size.

Tools of Data Collection: Data are collected using the following two tools

\section{Tool (I): Structured Interviewing Questionnaire:}

This tool is developed by the investigator and is composed of three main parts. The first part is developed to collect data related to socio-demographic characteristic of textile factory workers: such as age, family numbers, marital status, level of education and monthly income. The second part includes questions related to work characteristics: such as work shifts, daily working hours, years of experience, and position during work. The third part includes questions about types, sources and effects of occupational hazards on the worker's health. Tool (II): An observational checklist is developed by Hagag, (1995) to assess workers use for different personal protective clothes such as (overall uniform, head cover, eye goggles, gloves, protective apron, safety boots and respiratory mask).

\section{Tool Content Validity and Reliability:}

The Structured Interviewing Questionnaire was developed by the researcher and it was tested for content validity by a panel of five experts in the field of community health nursing and public health medicine. The reliability was assured by calculating cronbach's alpha coefficients for each factor, it was high (0.88).

\section{Pilot study:}

The pilot study was carried out with $10 \%$ of study sample which included (12) workers, who were selected randomly from textile factory in Damietta city. It was done to ascertain the relevance, clarity \& applicability of the developed tool and to estimate the time needed to fill the questionnaire sheet. Those workers who shared in the pilot study were excluded from the main study sample as a result of the modifications made to the questionnaire sheet, where some of the questions were added and others were omitted and rewording. The final form of the tool was formulated and the time needed for completing them was also determined. 


\section{Administrative and Ethical Considerations:}

An official permission was obtained by submission of an official letter from the Faculty of Nursing to the responsible authorities of the study setting to obtain the authorization for data collection. The aim of the study was explained to every worker before participation, and voluntary participation was emphasized and an oral consent was obtained.

\section{Statistical Analysis}

Data entry and statistical analysis were done using SPSS 16.0 statistical software package. Data were presented using descriptive statistics in the form of frequencies and percentages for qualitative variables, and means and standard deviations for quantitative variables. Qualitative categorical variables were compared using chi-square test. Whenever the expected values in one or more of the cells in a $2 \times 2$ tables was less than 5, Fisher exact test and Monte Carlo was used instead.

\section{Field Work:}

After obtaining the official permission to conduct the study and after finalization of tools, the investigator met with the workers individually and explained to them the purpose of the study, the consent of each eligible workers were obtained before their participation. Data collection period extended two months from the beginning of November to the end of December 2014, during the morning and afternoon shifts for three days per week. Time needed for filling Questionnaire ranged from 20 to 30 minutes. Observational checklist was carried out by the investigator, during morning and afternoon shifts for three days per week, with the average 1-2 workers per day and 3-6 workers per week.

\section{RESULTS:}

Table (1): shows that the age of the study group ranged from 18- 60 years with a mean age $39.07 \pm 12.63$ years and two-fifths $(40.0 \%)$ of them were between the ages of 46-60 years. Concerning marital status, it was found that the highest percentages $(59.3 \%)$ of the study group were married and the lowest percentages $(2.8 \%)$ were divorced. Regarding to level of education, it was observed that slightly less than twothirds $(63.9 \%)$ of the study group had a technical secondary education, while $2.8 \%$ of them only had basic education. In relation to crowding index, the table revealed that the mean crowding index was $1.58 \pm 0.56$. Eventually, the table explained that slightly 
more than two-thirds $(66.7 \%)$ of the study group reported that each hadn't enough income.

Table (2): demonstrats that less than half (46.3\%) of the study group are working morning shifts, slightly more than one-fifth $(21.3 \%)$ have been working more than one shift, and slightly less than one-third (32.4\%) are working on afternoon shifts. The results also revealed that the working hours of the study group are ranged between $6-12$ hours per day with a median of eight hours, more than half (52.8\%) are working less or equal to eight hours per day, while those who work more than eight hours per day represented $47.2 \%$. In relation to years of experience, half (50.0\%) of the study group reported having experience more than 10 years, while only $7.4 \%$ of the study group reported having experience less than or equal one year. As regards to position of the study group during working, less than half (46.3\%) of them are working in a standing position, while those who work in a sitting and bending positions are $39.8 \%$ and $13.9 \%$, respectively (as they reported).

Table (3): reveales that $71.3 \%$ of the study group are exposed to physical hazards, $69.4 \%$ are exposed to chemical hazards, while $66.7 \%$ \& $59.3 \%$ are exposed to mechanical and psychological hazards respectively.

Table (4): detects that slightly more than three quarters $(76.6 \%)$ of the study group are exposed to noisy hazard; more than half $(55.8 \%)$ of them are exposed to extreme temperature, while those who exposed to vibration and electrical shock are in the following percentages $32.5 \%$ and $20.8 \%$ respectively. Regarding to health problems resulting from exposure to physical hazards, it was found that the majority of the study group (88.3\%) had health problems such as headache, hearing problems, and electrical shocks in percentages of $66.2 \%, 55.9 \%$, and $14.7 \%$ respectively.

Table (5): displays that $69.4 \%$ of the study group are exposed to different sources of chemical hazards such as cotton dust, irritant chemicals and mineral oils, vapors and fumes in percentages of $93.3 \%, 34.7 \%$, and $14.7 \%$ respectively. Regarding to health problems due to exposure to chemical hazards, it was found, that the majority $(81.3 \%)$ of the study group had health problems, the highest percentage $(96.7 \%)$ of them had respiratory tract diseases, followed by chest and Eye allergy which represented $60.7 \%$ for each, while those who had nose allergy due to exposure to chemical hazards were 
$59.0 \%$, and the least percentage $(32.8 \%)$ of them had skin disorders (cracked and dry skin).

Table (6): illustrates that $66.7 \%$ of the study group are exposed to different sources of mechanical hazards such as, prolonged standing, prolonged sitting, bending for long times, heavy lifting, falling/slipping and sudden movements in the following percentages: $83.3 \%, 68.0 \%, 50.00 \%, 50.00 \%, 34.7 \%$ and $31.9 \%$ respectively. As for health problems from exposure to mechanical hazards, it was found that most $(88.9 \%)$ of the study group had health problems such as low back pain, varicose and neck pain ( 79.2\%, 55.0\%, $34.7 \%$, respectively); while the lowest percentages among workers who had health problems like fractures, tears of ligaments, and strains $11.1 \%, 11.1 \%$ and $23.6 \%$ respectively.

Table (7): indicates that $59.3 \%$ of the study group are exposed to different sources of psychological hazards such as increased work load, length of work (overtime), lack of worker cooperation, verbal violence and lack of appreciation of supervisors $93.8 \%$, $71.9 \%, 68.8 \%, 62.5 \%$ and $31.3 \%$ respectively. Concerning the health problems resulting from exposure to psychological hazards, it was found that $89.1 \%$ of the study group had health problems such as stress and tension, job dissatisfaction and frequent absenteeism $94.7 \%, 87.8 \%$ and $33.3 \%$ respectively.

Table (8): reveales that $74.1 \%$ of the overall uniform were unavailable, $84.3 \%$ of the study group did not use head cover and $24.1 \%$ of the them were using the protective apron.

Table(9): accentuates that, there was a statistically significant difference between level of education of the study group and their exposure to various types of occupational hazards at the workplace, while there was no statistically significant differences between age, work duration, nor daily working hours of the study group with different types of occupational health hazard.

Table (10): shows that, there was no statistically significant difference between personal protective equipment and various types of occupational health hazards among the study group in the textile factory. 
Table (1): Distribution of the study group according to their socio demographic data $(\mathrm{n}=108)$

\begin{tabular}{|c|c|c|}
\hline Items & No. & $\%$ \\
\hline Age (years) & & \\
\hline$\leq 30$ & 32 & 29.6 \\
\hline $31-45$ & 33 & 30.4 \\
\hline $46-60$ & 43 & 40.0 \\
\hline Min. - Max. & \multirow{3}{*}{\multicolumn{2}{|c|}{$\begin{array}{c}18.0-60.0 \\
39.07 \pm 12.63 \\
38.0\end{array}$}} \\
\hline Mean \pm SD & & \\
\hline Median & & \\
\hline \multicolumn{3}{|l|}{ Marital status } \\
\hline Single & 24 & 22.2 \\
\hline Married & 64 & 59.3 \\
\hline Divorced & 3 & 2.8 \\
\hline Widowed & 17 & 15.8 \\
\hline \multicolumn{3}{|l|}{ Education } \\
\hline cannot read \& write & 10 & 9.3 \\
\hline Read and write & 13 & 12.0 \\
\hline Basic education & 3 & 2.8 \\
\hline Secondary education & 7 & 6.5 \\
\hline Technical secondary education & 69 & 63.9 \\
\hline University education & 6 & 5.6 \\
\hline \multicolumn{3}{|l|}{ Crowding index } \\
\hline Range. & \multicolumn{2}{|c|}{$0.33-3.0$} \\
\hline Mean \pm SD & \multicolumn{2}{|c|}{$1.58 \pm 0.56$} \\
\hline Median & \multicolumn{2}{|c|}{1.67} \\
\hline \multicolumn{3}{|l|}{ Monthly income } \\
\hline Not enough & 72 & 66.7 \\
\hline Enough & 36 & 33.3 \\
\hline Enough and more & - & - \\
\hline
\end{tabular}


Table (2): Distribution of the study group according to their work characteristics $(\mathrm{n}=108)$

\begin{tabular}{|c|c|c|}
\hline & No. & $\%$ \\
\hline Work Shifts & & \\
\hline Morning & 50 & 46.3 \\
\hline Afternoon & 35 & 32.4 \\
\hline More than one shift & 23 & 21.3 \\
\hline Working Hours / Day & & \\
\hline$\leq 8$ hours & 57 & 52.8 \\
\hline$>8$ hours & 51 & 47.2 \\
\hline Min. - Max. & \multirow{3}{*}{\multicolumn{2}{|c|}{$\begin{array}{c}6.0-12.0 \\
9.0 \pm 2.56 \\
8.0\end{array}$}} \\
\hline Mean \pm SD & & \\
\hline Median & & \\
\hline \multicolumn{3}{|l|}{ Working duration (years of experience) } \\
\hline$\leq 1$ & 8 & 7.4 \\
\hline $2-\leq 5$ & 20 & 18.5 \\
\hline $6-\leq 10$ & 26 & 24.1 \\
\hline$>10$ & 54 & 50.0 \\
\hline Min. - Max. & \multirow{3}{*}{\multicolumn{2}{|c|}{$\begin{array}{c}1.0-37.0 \\
14.05 \pm 10.73 \\
10.50\end{array}$}} \\
\hline Mean \pm SD & & \\
\hline Median & & \\
\hline Position during Work & & \\
\hline Sitting & 43 & 39.8 \\
\hline Standing & 50 & 46.3 \\
\hline Bending for long periods & 15 & 13.9 \\
\hline
\end{tabular}

Table (3): Distribution of the study group according to their exposure to occupational health hazards (as they reported).

\begin{tabular}{|l|c|c|c|c|}
\hline \multirow{2}{*}{\multicolumn{1}{c|}{ Occupational Hazards }} & \multicolumn{2}{c|}{ Exposed } & \multicolumn{2}{c|}{ Not exposed } \\
\cline { 2 - 5 } & NO & \% & NO & \% \\
\hline Physical hazards & 77 & 71.3 & 31 & 28.7 \\
Chemical hazards & 75 & 69.4 & 33 & 30.6 \\
Mechanical hazards & 72 & 66.7 & 36 & 33.3 \\
Psychological hazards & 64 & 59.3 & 44 & 40.7 \\
\hline
\end{tabular}

Responses are not mutually exclusive 
Table (4): Distribution of the study group according to their exposure to sources of physical hazard as reported by them. $(n=77)$.

\begin{tabular}{|l|c|c|}
\hline \multicolumn{2}{|c|}{ Sources of Physical Hazards } & \multicolumn{2}{c|}{ Exposed Workers } \\
\cline { 2 - 3 } & $\mathbf{( N = 7 7 )}$ & $\mathbf{( 7 1 . 3 \% )}$ \\
\hline Exposure to: & & \\
Noise & 59 & 76.6 \\
Extreme Temperature & 43 & 55.8 \\
Vibrations & 25 & 32.5 \\
Radiation & 0 & 0.0 \\
Electrical Shock & 16 & 20.8 \\
\hline Effects on the workers' health: & & \\
Exposed without effect on the workers 'health & 9 & 11.7 \\
\hline Exposed with health problems: & 68 & 88.3 \\
Hearing problems & 38 & 55.9 \\
Electrical shock & 10 & 14.7 \\
Headache & 45 & 66.2 \\
\hline
\end{tabular}

Responses are not mutually exclusive

Table (5): Distribution of the study group according to their exposure to chemical hazards as reported by them. $(n=75)$.

\begin{tabular}{|l|c|c|}
\hline \multirow{2}{*}{ Sources of Chemical Hazards } & \multicolumn{2}{|c|}{ Exposed workers } \\
\cline { 2 - 3 } & $\mathbf{( N = 7 5 )}$ & $\mathbf{6 9 . 4 \%}$ \\
\hline Exposure to: & 70 & 93.3 \\
Cotton dust & 11 & 14.7 \\
Fumes and vapors & 26 & 34.7 \\
Irritant chemicals and minerals oil & & \\
\hline Effects on the workers' health: & 14 & 18.7 \\
Exposed without effect on the workers' health & & \\
& 61 & 81.3 \\
Exposed with health problems : & 37 & 60.7 \\
Chest allergy & 36 & 59.0 \\
Nose allergy & 59 & 96.7 \\
Respiratory tract diseases (pneumonia) & 37 & 60.7 \\
Eye allergy & 20 & 32.8 \\
Skin disorder( cracked and dry skin) & & \\
\hline
\end{tabular}

Response are not mutually exclusive 
Table (6): Distribution of the study group according to their exposure to mechanical hazards as reported by them. $(\mathrm{n}=72)$.

\begin{tabular}{|l|c|c|}
\hline \multirow{2}{*}{ Sources of Mechanical Hazards } & \multicolumn{2}{|c|}{ Exposed workers } \\
\cline { 2 - 3 } & (n=72) & $\mathbf{6 6 . 7 \%}$ \\
\hline Exposure to: & 36 & 50.0 \\
Heavy lifting & 60 & 83.3 \\
Prolonged standing & 49 & 68.0 \\
Prolonged sitting & 25 & 34.7 \\
Falling/ slipping & 23 & 31.9 \\
Sudden movement & 36 & 50.0 \\
Bending for a long time & & \\
\hline Effects on the workers' health: & 8 & 11.1 \\
Exposed without effect on the workers' health & & \\
& 64 & 88.9 \\
Exposed with health problems: & 40 & 55.5 \\
Varicose veins & 57 & 79.2 \\
Low back pain & 17 & 23.6 \\
Strains & 8 & 11.1 \\
Fractures & 25 & 34.7 \\
Neck pain & 8 & 11.1 \\
Tears of leg ligaments & & \\
\hline
\end{tabular}

Response are not mutually exclusive.

Table (7): Distribution of the study group according to their exposure to psychological health hazards as reported by them. $(n=64)$

\begin{tabular}{|l|c|c|}
\hline \multirow{2}{*}{ Sources of Psychological Hazards } & \multicolumn{2}{|c|}{ Exposed Workers } \\
\cline { 2 - 3 } & $\mathbf{( n = 6 4 )}$ & $\mathbf{5 9 . 3 \%}$ \\
\hline Exposure to: & 40 & 62.5 \\
Verbal violence & 46 & 71.9 \\
Length of work (over time ) & 20 & 31.3 \\
Lack of appreciation from supervisors & 60 & 93.8 \\
Increased work load & 44 & 68.8 \\
Lack of workers co- operation & & \\
\hline Effects on the workers' health: & 7 & 10.9 \\
Exposed without effect on the workers' health & 7 & 89.1 \\
Exposed with health problems: & 54 & 94.7 \\
Stress and tension & 19 & 33.3 \\
Frequent absenteeism & 50 & 87.8 \\
Job dissatisfaction & & \\
\hline
\end{tabular}

Response are not mutually exclusive. 
Table (8): Distribution of the study group according to their using to personal protective equipment $(\mathrm{n}=108)$.

\begin{tabular}{|c|c|c|}
\hline \multirow[b]{2}{*}{ Personal protective equipment } & \multicolumn{2}{|c|}{$(n=108)$} \\
\hline & No. & $\%$ \\
\hline $\begin{array}{l}\text { Overall uniform } \\
\text { Not available } \\
\text { Not used } \\
\text { Used }\end{array}$ & $\begin{array}{c}80 \\
27 \\
1\end{array}$ & $\begin{array}{c}74.1 \\
25.0 \\
0.9\end{array}$ \\
\hline $\begin{array}{l}\text { Head cover } \\
\text { Not available } \\
\text { Not used } \\
\text { Used }\end{array}$ & $\begin{array}{c}6 \\
91 \\
11\end{array}$ & $\begin{array}{c}5.6 \\
84.3 \\
10.2\end{array}$ \\
\hline $\begin{array}{l}\text { Eye goggles } \\
\text { Not available } \\
\text { Not used } \\
\text { Used }\end{array}$ & $\begin{array}{c}71 \\
33 \\
4\end{array}$ & $\begin{array}{c}65.7 \\
30.6 \\
3.7\end{array}$ \\
\hline $\begin{array}{l}\text { Gloves } \\
\text { Not available } \\
\text { Not used } \\
\text { Used }\end{array}$ & $\begin{array}{c}1 \\
89 \\
18\end{array}$ & $\begin{array}{c}0.9 \\
82.4 \\
16.7\end{array}$ \\
\hline $\begin{array}{l}\text { Protective apron } \\
\text { Not available } \\
\text { Not used } \\
\text { Used }\end{array}$ & $\begin{array}{c}3 \\
79 \\
26\end{array}$ & $\begin{array}{c}2.8 \\
73.1 \\
24.1\end{array}$ \\
\hline $\begin{array}{l}\text { Safety boots } \\
\text { Not available } \\
\text { Not used } \\
\text { Used }\end{array}$ & $\begin{array}{c}2 \\
90 \\
16\end{array}$ & $\begin{array}{c}1.9 \\
83.3 \\
14.8\end{array}$ \\
\hline $\begin{array}{l}\text { Respiratory mask } \\
\text { Not available } \\
\text { Not used } \\
\text { Used }\end{array}$ & $\begin{array}{c}2 \\
88 \\
18\end{array}$ & $\begin{array}{c}1.9 \\
81.5 \\
16.7\end{array}$ \\
\hline
\end{tabular}

\section{$\chi^{2}:$ Chi square test for Friedman test}

$*$ : Statistically significant at $p \leq 0.05$ 
Table (9): Relationship between personal and work characteristics of the study group with occupational hazards

\begin{tabular}{|c|c|c|c|c|c|c|c|c|c|c|c|c|c|c|c|c|}
\hline & \multicolumn{4}{|c|}{ Physical hazards } & \multicolumn{4}{|c|}{ Chemical hazards } & \multicolumn{4}{|c|}{ Mechanical hazards } & \multicolumn{4}{|c|}{ Psychological hazards } \\
\hline & \multicolumn{2}{|c|}{$\begin{array}{c}\text { Not exposed } \\
(\mathrm{n}=31)\end{array}$} & \multicolumn{2}{|c|}{$\begin{array}{c}\text { Exposed } \\
(n=77)\end{array}$} & \multicolumn{2}{|c|}{$\begin{array}{c}\text { Not exposed } \\
(n=33)\end{array}$} & \multicolumn{2}{|c|}{$\begin{array}{c}\text { Exposed } \\
(n=75)\end{array}$} & \multicolumn{2}{|c|}{$\begin{array}{c}\text { Not exposed } \\
(n=36)\end{array}$} & \multicolumn{2}{|c|}{$\begin{array}{c}\text { Exposed } \\
(n=72)\end{array}$} & \multicolumn{2}{|c|}{$\begin{array}{c}\text { Not exposed } \\
(n=44)\end{array}$} & \multicolumn{2}{|c|}{$\begin{array}{c}\text { Exposed } \\
(n=64)\end{array}$} \\
\hline & No & $\%$ & No & $\%$ & No & $\%$ & No & $\%$ & No & $\%$ & No & $\%$ & No & $\%$ & No & $\%$ \\
\hline Age (years) & & & & & & & & & & & & & & & & \\
\hline$\leq 30$ & 6 & 19.4 & 26 & 33.8 & 13 & 39.4 & 19 & 25.3 & 13 & 36.1 & 19 & 26.4 & 15 & 34.1 & 17 & 26.6 \\
\hline $31-45$ & 11 & 35.5 & 22 & 28.6 & 10 & 30.3 & 23 & 30.7 & 10 & 27.8 & 23 & 31.9 & 13 & 29.5 & 20 & 31.3 \\
\hline $46-60$ & 14 & 45.2 & 29 & 37.7 & 10 & 30.3 & 33 & 44.0 & 13 & 36.1 & 30 & 41.7 & 16 & 36.4 & 27 & 42.2 \\
\hline$\square^{\square} \square \square \mathbf{p} \square$ & \multicolumn{4}{|c|}{$2.207(0.332)$} & \multicolumn{4}{|c|}{$2.610(0.271)$} & \multicolumn{4}{|c|}{$1.088(0.580)$} & \multicolumn{4}{|c|}{$0.746(0.689)$} \\
\hline Working dur & & & & & & & & & & & & & & & & \\
\hline$\leq 1$ & 2 & 6.5 & 6 & 7.8 & 2 & 6.1 & 6 & 8.0 & 2 & 5.6 & 6 & 8.3 & 2 & 4.5 & 6 & 9.4 \\
\hline $2-\leq 5$ & 6 & 19.4 & 14 & 18.2 & 8 & 24.2 & 12 & 16.0 & 8 & 22.2 & 12 & 16.7 & 10 & 22.7 & 10 & 15.6 \\
\hline $6-\leq 10$ & 5 & 16.1 & 21 & 27.3 & 10 & 30.3 & 16 & 21.3 & 10 & 27.8 & 16 & 22.2 & 11 & 25.0 & 15 & 23.4 \\
\hline$>10$ & 18 & 58.1 & 36 & 46.8 & 13 & 39.4 & 41 & 54.7 & 16 & 44.4 & 38 & 52.8 & 21 & 47.7 & 33 & 51.6 \\
\hline$\square^{\square} \square \square \mathbf{p} \square$ & \multicolumn{4}{|c|}{$1.776(0.620)$} & \multicolumn{4}{|c|}{$2.792(0.425)$} & \multicolumn{4}{|c|}{$1.291(0.731)$} & \multicolumn{4}{|c|}{$1.592\left({ }^{\mathrm{MC}} \mathrm{p}=0.682\right)$} \\
\hline Education & & & & & & & & & & & & & & & & \\
\hline Cannot read \& write & 4 & 12.9 & 6 & 7.8 & 1 & 3.0 & 9 & 12.0 & 1 & 2.8 & 9 & 12.5 & 2 & 4.5 & 8 & 12.5 \\
\hline Read and write & 5 & 16.1 & 8 & 10.4 & 1 & 3.0 & 12 & 16.0 & 1 & 2.8 & 12 & 16.7 & 3 & 6.8 & 10 & 15.6 \\
\hline Basic education & 4 & 12.9 & 3 & 3.9 & 1 & 3.0 & 6 & 8.0 & 1 & 2.8 & 6 & 8.3 & 2 & 4.5 & 5 & 7.8 \\
\hline Secondary education & 0 & 0.0 & 3 & 3.9 & 1 & 3.0 & 2 & 2.7 & 1 & 2.8 & 2 & 2.8 & 1 & 2.3 & 2 & 3.1 \\
\hline Technical secondary education & 17 & 54.8 & 52 & 67.5 & 25 & 75.8 & 44 & 58.7 & 28 & 77.8 & 41 & 56.9 & 31 & 70.5 & 38 & 59.4 \\
\hline University education & 1 & 3.2 & 5 & 6.5 & 4 & 12.1 & 2 & 2.7 & 4 & 11.1 & 2 & 2.8 & 5 & 11.4 & 1 & 1.6 \\
\hline$\square^{\square} \square \square^{\mathbf{M C}} \mathbf{p} \square$ & \multicolumn{4}{|c|}{$9.323^{*}\left(0.004^{*}\right)$} & \multicolumn{4}{|c|}{$10.299^{*}\left(0.046^{*}\right)$} & \multicolumn{4}{|c|}{$11.768^{*}\left(0.023^{*}\right)$} & \multicolumn{4}{|c|}{$9.463\left(0.007^{*}\right)$} \\
\hline \multicolumn{17}{|l|}{ Working Hours / Day } \\
\hline More than 8 hours & 22 & 71.0 & 35 & 45.5 & 17 & 51.5 & 40 & 53.3 & 17 & 47.2 & 40 & 55.6 & 19 & 43.2 & 38 & 59.4 \\
\hline Less than 8 hours & 9 & 29.0 & 42 & 54.5 & 16 & 48.5 & 35 & 46.7 & 19 & 52.8 & 32 & 44.4 & 25 & 56.8 & 26 & 40.6 \\
\hline$\square^{\square} \square \square \mathbf{p} \square$ & \multicolumn{4}{|c|}{$4.065(0.114)$} & \multicolumn{4}{|c|}{$0.030(0.862)$} & \multicolumn{4}{|c|}{$0.669(0.413)$} & & 2.74 & 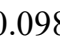 & \\
\hline
\end{tabular}

$\chi^{2}$ : Chi square testMC: Monte Carlo for Chi square test*: Statistically significant at $p$ $\leq 0.05$ 
Table(10): Relationship between occupational health hazards and different types of personal protective equipment

\begin{tabular}{|c|c|c|c|c|c|c|c|c|c|c|c|c|c|c|c|c|}
\hline & \multicolumn{4}{|c|}{ Physical hazards } & \multicolumn{4}{|c|}{ Chemical hazards } & \multicolumn{4}{|c|}{ Mechanical hazards } & \multicolumn{4}{|c|}{ Psychological hazards } \\
\hline & \multicolumn{2}{|c|}{$\begin{array}{c}\text { Not exposed } \\
\quad(n=31)\end{array}$} & \multicolumn{2}{|c|}{$\begin{array}{c}\text { Exposed } \\
(n=77)\end{array}$} & \multicolumn{2}{|c|}{$\begin{array}{l}\text { Not exposed } \\
\quad(n=33)\end{array}$} & \multicolumn{2}{|c|}{$\begin{array}{c}\text { Exposed } \\
(n=75)\end{array}$} & \multicolumn{2}{|c|}{$\begin{array}{c}\text { Not exposed } \\
\quad(n=36)\end{array}$} & \multicolumn{2}{|c|}{$\begin{array}{c}\text { Exposed } \\
(n=72)\end{array}$} & \multicolumn{2}{|c|}{$\begin{array}{l}\text { Not exposed } \\
(n=44)\end{array}$} & \multicolumn{2}{|c|}{$\begin{array}{c}\text { Exposed } \\
(n=64)\end{array}$} \\
\hline & No & $\%$ & No & $\%$ & No & $\%$ & No & $\%$ & No & $\%$ & No & $\%$ & No & $\%$ & No & $\%$ \\
\hline Overall uniform & & & & & & & & & & & & & & & & \\
\hline Not available + & 22 & 71.0 & 58 & 75.3 & 21 & 63.6 & 59 & 78.7 & 25 & 69.4 & 55 & 76.4 & 32 & 72.7 & 48 & 75.0 \\
\hline Not used + & 9 & 29.0 & 18 & 23.4 & 11 & 33.3 & 16 & 21.3 & 10 & 27.8 & 17 & 23.6 & 11 & 25.0 & 16 & 25.0 \\
\hline Used & 0 & 0.0 & 1 & 1.3 & 1 & 3.0 & 0 & 0.0 & 1 & 2.8 & 0 & 0.0 & 1 & 2.3 & 0 & 0.0 \\
\hline$\square^{\square} \square \square^{\mathbf{M C}} \mathbf{p} \square$ & \multicolumn{4}{|c|}{$0.837(0.733)$} & \multicolumn{4}{|c|}{$4.116(0.109)$} & \multicolumn{4}{|c|}{$2.222(0.370)$} & \multicolumn{4}{|c|}{$1.413(0.627)$} \\
\hline Head cover & & & & & & & & & & & & & & & & \\
\hline Not available + & 0 & 0.0 & 6 & 7.8 & 5 & 15.2 & 1 & 1.3 & 5 & 13.9 & 1 & 1.4 & 6 & 13.6 & 0 & 0.0 \\
\hline Not used + & 26 & 83.9 & 65 & 84.4 & 23 & 69.7 & 68 & 90.7 & 26 & 72.2 & 65 & 90.3 & 33 & 75.0 & 58 & 90.6 \\
\hline Used & 5 & 16.1 & 6 & 7.8 & 5 & 15.2 & 6 & 8.0 & 5 & 13.9 & 6 & 8.3 & 5 & 11.4 & 6 & 9.4 \\
\hline$\square^{\square} \square \square^{\mathbf{M C}} \mathbf{p} \square$ & \multicolumn{4}{|c|}{$3.637(0.146)$} & \multicolumn{4}{|c|}{$5.677(0.313)$} & \multicolumn{4}{|c|}{$7.898(0.417)$} & \multicolumn{4}{|c|}{$\frac{1}{8.493(0.108)}$} \\
\hline Eye goggles & & & & & & & & & & & & & & & & \\
\hline Not available + & 15 & 48.4 & 56 & 72.7 & 27 & 81.8 & 44 & 58.7 & 30 & 83.3 & 41 & 56.9 & 36 & 81.8 & 35 & 54.7 \\
\hline Not used + & 14 & 45.2 & 19 & 24.7 & 6 & 18.2 & 27 & 36.0 & 6 & 16.7 & 27 & 37.5 & 8 & 18.2 & 25 & 39.1 \\
\hline Used & 2 & 6.5 & 2 & 2.6 & 0 & 0.0 & 4 & 5.3 & 0 & 0.0 & 4 & 5.6 & 0 & 0.0 & 4 & 6.3 \\
\hline$\square^{\square} \square \square^{\mathbf{M C}} \mathbf{p} \square$ & \multicolumn{4}{|c|}{$6.152(0.079)$} & \multicolumn{4}{|c|}{ 5.444(0.058) } & & 7.488 & $\overline{093)}$ & & & 9.7 & $\overline{(0.30}$ & \\
\hline Gloves & & & & & & & & & & & & & & & & \\
\hline Not available + & 1 & 3.2 & 0 & 0.0 & 0 & 0.0 & 1 & 1.3 & 0 & 0.0 & 1 & 1.4 & 0 & 0.0 & 1 & 1.6 \\
\hline Not used + & 26 & 83.9 & 63 & 81.8 & 24 & 72.7 & 65 & 86.7 & 27 & 75.0 & 62 & 86.1 & 34 & 77.3 & 55 & 85.9 \\
\hline Used & 4 & 12.9 & 14 & 18.2 & 9 & 27.3 & 9 & 12.0 & 9 & 25.0 & 9 & 12.5 & 10 & 22.7 & 8 & 12.5 \\
\hline$\square^{\square} \square \square^{\mathbf{M C}} \mathbf{p} \square$ & & 2.532 & .327 & & & 5.776 & .116 & & & 3.059 & 200 & & & 2.4 & $(0.23$ & \\
\hline Protective apron & & & & & & & & & & & & & & & & \\
\hline Not available + & 0 & 0.0 & 3 & 3.9 & 3 & 9.1 & 0 & 0.0 & 3 & 8.3 & 0 & 0.0 & 3 & 6.8 & 0 & 0.0 \\
\hline Not used + & 24 & 77.4 & 55 & 71.4 & 22 & 66.7 & 57 & 76.0 & 23 & 63.9 & 56 & 77.8 & 29 & 65.9 & 50 & 78.1 \\
\hline Used & 7 & 22.6 & 19 & 24.7 & 8 & 24.2 & 18 & 24.0 & 10 & 27.8 & 16 & 22.2 & 12 & 27.3 & 14 & 21.9 \\
\hline $\operatorname{lup}^{\square} \square^{\mathbf{M C}} \mathbf{p} \square$ & & 0.850 & 0.690 & & & 5.981 & .052 & & & 6.120 & .241 & & & & $(0.08$ & \\
\hline Safety boots & & & & & & & & & & & & & & & & \\
\hline Not available + & 0 & 0.0 & 2 & 2.6 & 1 & 3.0 & 1 & 1.3 & 1 & 2.8 & 1 & 1.4 & 1 & 2.3 & 1 & 1.6 \\
\hline Not used + & 25 & 80.6 & 65 & 84.4 & 28 & 84.8 & 62 & 82.7 & 30 & 83.3 & 60 & 83.3 & 37 & 84.1 & 53 & 82.8 \\
\hline Used & 6 & 19.4 & 10 & 13.0 & 4 & 12.1 & 12 & 16.0 & 5 & 13.9 & 11 & 15.3 & 6 & 13.6 & 10 & 15.6 \\
\hline$\square^{\square} \square \square^{\mathbf{M C}} \mathbf{p} \square$ & & 1.172 & .580 & & & 0.921 & 690 & & & 0.632 & .000 & & & 0.4 & $(1.00$ & \\
\hline Respiratory mask & & & & & & & & & & & & & & & & \\
\hline Not available + & 0 & 0.0 & 2 & 2.6 & 1 & 3.0 & 1 & 1.3 & 1 & 2.8 & 1 & 1.4 & 1 & 2.3 & 1 & 1.6 \\
\hline Not used + & 24 & 77.4 & 64 & 83.1 & 26 & 78.8 & 62 & 82.7 & 29 & 80.6 & 59 & 81.9 & 35 & 79.5 & 53 & 82.8 \\
\hline Used & 7 & 22.6 & 11 & 14.3 & 6 & 18.2 & 12 & 16.0 & 6 & 16.7 & 12 & 16.7 & 8 & 18.2 & 10 & 15.6 \\
\hline$\square^{\square} \square \square^{\mathbf{M C}} \mathbf{p} \square$ & & 1.508 & .436 & & & 0.901 & 698 & & & 0.628 & .000 & & & 0.5 & $(0.89$ & \\
\hline
\end{tabular}

$\chi^{2}$ : chi square test MC: Monte Carlo Square test: Statistically significant at $\mathrm{p} \leq 0.05$ 


\section{DISCUSSION:}

Textile industry is one of labor intensive production and most technologically complex of all industries, and is a place of work where workers are exposed to different safety hazards, like cotton dust, excessive noise, accidents and diseases. As result of this, workers, employers and government lost direct and indirect costs related to workplace injuries and illnesses. The direct costs for employers include compensation and treatment costs that has to be paid to the disabled workers, and while the indirect costs include production disturbance costs, lost time of injured worker, time lost by supervisors or executive to follow the injured worker, training costs for new workers. The direct costs for workers include pain and suffering from the injury or illness, loss of income, loss of a job and health-care costs, and while the indirect costs include time lost by family members to care the disabled worker and utmost economic shock and social chaos (Tadesse et al., 2016).

Concerning the sociodemographic data of the study group, it was shown that the age of the study group ranged from 18- 60 years with a mean age $39.07 \pm 12.63$ years and two-fifths of them were between the ages of 46-60 years, the highest percentage were married and the lowest percentages were divorced, slightly less than two-thirds had a technical secondary education and slightly more than two-thirds of the study group reported that each hadn't enough income.

Regarding exposure of the study group to several cases of occupational health hazards at the workplace, the current study revealed that less than three quarters of the study group is exposed to physical risks, slightly more than two thirds are exposed to chemical hazards, two thirds are exposed to mechanical hazards and more than half of the study group are exposed to psychological hazards. These findings might be due to, lack of awareness about the risks of their occupations, general backwardness in sanitation, poor nutrition, lack of orientation around the universal precautions of the occupational health hazards in addition to lack of training program attendance.

These results are supported by findings of researches of Koka $\&$ Srivastava (2012); Marmot \& Blatt (2013), that the workers expose to various types of occupational health hazards in their workplace such as physical, chemical, mechanical and psychological hazards. In addition, Rantanen (2004) who conducted a survey about health and safety 
workplace, revealed that still $20 \%-30 \%$ of the actors in the industrialized countries, and up $50-70 \%$ of the working masses in producing nations are exposed to traditional physical-chemical, ergonomic and safety risks

In relation to exposure of the study group to sources of physical hazards, the present study indicated that slightly more than three quarters of whom exposed to physical risks, were exposed to noise, more than half reported exposure to extreme temperature, one third told exposure to vibration and one fifth said that exposed to electrical shock. These findings might be due to inadequate maintenance and break up of machines, ineffective follow-up of rules and regulations, low availability of ear muffs, machines were also old and dropped dead, poor work security, stripped electrical wires of some automobiles, over crowdedness by workers.

These findings are on the same line with Haider et al. (2008) who carried out a study about noise induced hearing loss among The Textile Industry Workers and revealed that noise was one of the common physical and environmental risks in the workplace Moreover, Sheha (2009) who conducted a study about Occupational Hazards among Workers in Glass Manufactures Industries, reported that noise hazard had the highest share of physical hazard exposure, followed by extreme temperature exposure.

Touching to the effects of the previously mentioned physical hazards to the health by studying the group, it was found that approximately two thirds suffered from headache, more than half having hearing problems, and more than one tenth reported suffering from electrical shock. These findings come in line with Takele et al. (2008) who conducted a study about Prevalence and Burden of Primary Headache in Akaki Textile Mill Workers, Ethiopia and revealed that headache disorders were the most common complaints at workplaces. Moreover, Nahar et al. (2010) who conducted a study about occupational health hazards in garment sector indicated that the most frequent illness reported by 95.56 percent of workers was affected by headache. On the other hand, the subject area about health Concerns among Workers in Weaving Industry, which was conducted by Anjum et al. (2009), showed that headache caused by physical hazards represented only $28 \%$ of the total health problems that took place in the workplace. Also, Nirmala (2013), who carried a study about Workplace Hazards Faced by Workers in Textile Industry as a Part of Occupational Health and 
Safety, revealed that hearing problem was one of the most occurring health problems which can be hard to identify because the effects build up slowly with time.

Concerning exposure of the study group to chemical hazards in the workplace, the work demonstrated that, most of those who exposed to chemical hazards, were exposed to cotton dust, slightly more than one third were exposed to irritant chemicals and mineral oil and more than one tenth were exposed to fumes and vapors. These determinations, in agreement with Suryakar et al. (2010) who conducted a study about Oxidative Stress in Cotton Industry Workers from Solapur City, and divulged that the textile mill workers were continuously exposed to cotton dust, which might contribute to different respiratory disorders such as byssinosis, respiratory tract irritation, COPD. In addition, Abd El-Hameed et al. (2012) who conducted a study to evaluate Compliance with Personal Protective Equipment among Workers at Textile Industry indicated that cotton dust and irritant chemicals were of the most important and common hazards in textile manufacture. Moreover, Pohanish (2012) revealed that the occupational safety and health administration, had estimated that about a million workers were involved in work with cotton fibers and thus were potentially exposed to cotton dust in the work.

Considering the effects resulted from chemical hazards' exposure, the findings clarified that, most of the study group had respiratory tract diseases, less than two thirds suffered from chest and eye allergy, more than half had nose allergy, and nearly one third had skin disorders. These health problems might be resulted from a big quantity of cotton dusts that the study group was exposed during the textile industry process, different chemical substances that the study group used mainly in dyeing, printing and completing operations. These results in agreements with Mahmoud et al. (2004) who bore out a study about Occupational Health Hazards among the Workers in Assiut Spinning Factory, and indicated that the major health problems related with cotton dust were respiratory problems.

Moreover, Ajeet et al. (2010) who conducted a study to study the Prevalence of Chronic Respiratory Morbidities and Related Epidemiological Factors among Spinning Mill Workers concluded that the workers were at peril of suffering from various chronic respiratory illnesses like byssinosis, chronic bronchitis due to vulnerability to the cotton dust in the work. On the contrary, to the present findings 
United State Bureau of labor statistics showed that occupational skin diseases mostly in the form of allergic and irritant (contact) dermatitis are the second most common type of occupational diseases Laundy \& Janes (2007). Moreover, Malik (2010) reported that more than half of his studied groups were exposed to skin disorders.

As regards exposure of the study group to sources of mechanical hazards at the textile factory, the current study detected that, the majority of the studied group mentioned that, they were exposed to prolonged standing, slightly more than two thirds were in sitting positions, half was exposed to heavy lifting and bending for a long time, Slightly more than third were exposed to falling and skidding, and slightly one third were exposed to sudden movements.

As for the effects resulting from mechanical hazards, the present study had indicated that, the highest percentage of whom reported mechanical hazards' exposure, suffered from low back pain, more than half were suffering from varicose veins, more than one third suffering from neck pain, slightly less than a quarter were suffering from strains, and the lowest suffering from fractures and tear in ligaments, respectively .This result is in agreement with Abd El-Fatah (2013) who conducted a study about Nurse's Occupational Hazards at General Hospitals in Port Said City, and revealed that nurses were exposed to different sources of environmental/ mechanical hazards such as lifting, prolonged standing, poor lighting, inadequate ventilation, overcrowded work area, sudden movement and falling/ slipping, respectively. Also, Gaber (2009) in his study about Development of Risk Management Standards for Zagazig University Hospitals, reported that fatigue, prolonged standing, back pain, varicose veins had the highest percentage of environmental and ergonomics risks.

Moreover, Shafik \& Abed El-Mohsen (2012) who conducted a study about Health Promotion Program to Improve Health Workers in Torah Cement Factory, reported that almost two thirds of the studied workers complained from musculoskeletal disorders.

In respect to reported psychological hazards among the textile workers, the findings clarified that slightly less than two thirds got psychological hazards such as increased workload, length of work (overtime), lack of worker cooperation, verbal violence and 
lack of appreciation of supervisors .As regards to the effects of such psychological hazards to the study group health, the present study revealed that the majority of the study group had health problems such as stress and tension, job dissatisfaction and frequent absenteeism. This finding is in agreement with, Kortum (2011), who conducted a study about perceptions of psychological hazards, work related stress, and workplace priority risks in developing countries, concluded that psychological hazards and work related stress were mostly seen as interchangeable in terms of the source and effect and all participants perceived them as a concern in their work force.

Contrary to the present findings Koesterich, (2011) in Colorado State University indicated low -level of occupational stress. On my point of view, these results might be due to high job demands, low control, effort reward imbalance, in adequate and un supportive supervision, Length of work (over time), Lack of appreciation from supervisors, Increased workload, poor relationship with co-workers, poor communication, bad leader ship, conflicting demands at work and home, Having too much to do, working under time pressure, badly designed shift system, job in security, risk of injury or death, un predictable working hours, work on holidays, and lack participation in decision making.

Concerning the relationship between personal, work characteristics and various types of occupational health hazards, the study revealed that, there was a statistical significant difference between level of education of the studied group with various types of occupational hazards while there was no statistical significant difference between age, work duration, or daily working hours with various types of occupational health hazards. These findings are in agreement with Ibrahim (2014), who carried out a study about the relationship between occupational health hazards and the use of safety measures among workers in textile factory in Port Said City, demonstrated that there was a highly statistical significant difference between levels of education of the studied group with various types of occupational hazards. In addition, Lee (2007) revealed that workers must be assured of their right to a safe and healthy work environment also suggested that there was a need to provide workers with information, education and training so that they would know best how to protect themselves. 
Moreover, Abd EL-Fatah (2013) revealed that there was no statistical significance difference between exposure to biological and physical health hazards among nurses at Port Said city, Egypt, with age groups. As well clarified that there was no statistical difference between exposure to physical and biological hazards with nursing's years of experience.

In respect to the relationship between usage of personal protective equipment by the study group and exposure to various occupational health hazards, the study concluded that, there was no statistical significant difference between personal protective equipment and various types of occupational hazards. This finding in contrast with Sheha (2009) who indicated that, there was a highly significant difference between occupational diseases and not wearing personal protective equipment. Also, in another study, Kotb (2012) concluded that there was a highly significant complaint in workers who weren't using personal protective devices than those who were using it.

\section{CONCLUSION:}

Most of the study group is exposed to various types of occupational health hazards at their workplaces which resulted in tremendous harmful effects on their health; there was obvious shortage and un availability of personal protective equipment. Also, there was no statistically significant difference between personal protective equipment and exposure to various types of occupational health hazards at the textile factory.

\section{RECOMMENDATIONS:}

1- The need for stressing on the application of International Standard Occupational Health and Safety Assessment Series, in field work to improve occupational health and safety performance for the workers

2- Periodic check up for workers for early detection of occupational hazards to monitor the health status and early case finding.

3- Emphasizing on the importance and usefulness of personal protective equipment to be used in the right way and first aid for the promotion of personal fitness of the workers by health promotion programs.

4- Health education programs should be developed and carried out by the occupational health nurse to raise the awareness of the workers regarding the bad effect of 
occupational health hazards, importance of attending training programs, and the proper use of personal protective equipment.

\section{REFERENCES:}

Abd EL-Fatah, F . (2013): Nurse's Occupational hazards at governmental hospitals in Port Said City .Un Published Master Thesis. Faculty of nursing, Port Said University.

Abd EL-Hameed, H., Aly, H., and Abd El-Latif, O. (2012): An intervention study to evaluate compliance with personal protective equipment among workers at Textile industry. Journal of American Science; 8 (7): 117-121. (ISSN: 1545-1003). Available at: http:// www.jofamericanscience.org.

Ajeet S., Aniruddha, D., Meenal, K., et al. (2010): To Study the Prevalence of Chronic Respiratory Morbidities and Related Epidemiological Factors among Spinning Mill Workers. Global Journal of Health Science; 2(2): 111-116.

Anjum, A., Mann, A,. and Anjum, A. (2009): Health Concerns among Workers in Weaving Industry: A Case Study of Tehsil Faialabad, Pakistan. . Agric. Soc. Sci; 5(3): 106-108. Available at: http://www.fspublishers.org

Babu, B., Parande, A., Raghu, S. et al. (2007): Cotton Textile Processing: Waste Generation and Effluent Treatment. The Journal of Cotton Science; 11(3):141-153. Available at: http://journal.cotton.org, (c) The Cotton Foundation 2007.

Gaber, M. (2009): Development of Risk Management Standards for Zagazig University Hospitals .Unpublished Doctorate Thesis. Faculty of Nursing, Cairo University.

Guzys, D. and Petrie, E. ( 2014): An Introduction to Community and Primary Health Care. $1^{\text {st }}$ ed., U.S.A: Cambridge University Press; P323. ISBN: 1107513529, 9781107513525 .

Haggag, M. (1995): The Impact of Child's Labor on His Health Status in Alexandria, Doctoral Thesis, Faculty of Nursing, Alexandria University. 
Haider, M., Taous, A., Rahim, M., et al. (2008): Noise induced hearing loss among the textile industry workers. Bangladesh Journal of Otorhinolaryngology; 14(2):39-45.

Hasanbeigi, A. and Price, L. (2012): A review of energy use and energy efficiency technologies for the textile industry; 16 (6): 3648-3665. DOI: 10.1016/j.rser.2012.03.029.

Hughes, P. and Ferrett, E. (2016): International Health and Safety at Work: For the NEBOSH International General Certificate in Occupational Health and Safety. $3^{\text {rd }}$ ed., New York 10017: Routledge; P 3. ISBN: 1317556704, 9781317556701.

Hunt ,R. (2009:. Introduction to community based nursing. $4^{\text {th }}$ ed., U.S.A: Lippincott Williams \& Wilkins; P. 355.

Ibrahim, A. (2014): Relationship between Occupational Health Hazards and the Use of Safety Measures among Workers in Textile Factory in Port Said City. Un Published Master Thesis. Faculty of nursing, Port Said University.

Koesteric, M. (2011): Review, Assessment, and prioritization for an occupational health and Safety management system in a veterinary Teaching Hospital using ANSI/AIHA Standards .Un published Master Thesis University of Colorado state.

Koka, $V$. and Srivastava, M. (2012): Occupational health hazards of textile workers of Pali District. Asian Journal of home science; 7 (1): 152-155.

Kortum, E. (2011): Psychosocial Risks and Work- Related Stress in Developing Countries: A call for Research and Action in Policy Development .Thesis for the degree of Doctor of Philosophy. University of Nottingham.

Kotb, $\boldsymbol{W}$. (2012): Health hazards among workers in Kaha food factory in Kalubya governorate. Un Published Master Thesis faculty of nursing, Benha University.

Lai, P., and Christiani, D. ( 2014): Long term respiratory health effects in textile workers. Curr Opin Pulm; 19(2): 152-157. doi:10.1097/MCP.0b013e32835cee9a.

Lee, T. (2007): Implement Safe Work Procedures: Safety and Health Management System Best Answer to Reduce Accidents at the Workplace' in Borneo Post, Friday. $7^{\text {th }}$ ed., December 2007; P.15 
Lundy, K. and Janes, S. (2007): Community Health Nursing .Caring for Public Health. $1^{\text {st }}$ ed., London: Jones and Bartlett Publisher; P. 947, 949, 952.

Mahmud, T., Abd El-Maged, H., Alaa El-Din, S., et al. (2004): A study of Occupational Health Hazards among Assuit Spinning Factory Workers. Ass. Univ. Bull. Environ .Res. 7(1):131-139.

Malik, N. (2010): Perspective of Occupational Health and Safety in Textile Industry. Degree of Doctorate of Philosophy in Rural Sociology. Faisalabad, Pakistan.

Marmot, M. and Blatt, P. (2013): Health Promotion at work places in Tanzania. African newsletter of occupational health and safety. 23(1): 3-23

Mousa, K., Fouad, N. and El-Dein, S. (2014): Assessment of Knowledge and SelfReported Practices of Iron Mines' Workers about Pneumoconiosis in Baharia Oasis, Giza Governorate. Med. J. Cairo Univ; 82( 2): 23-29. . www.medicaljournalofcairouniversity.net

Nahar, N., Ali, R. and Begum, F. (2010): occupational health hazards in garment sector. int. j. bio res; 1(2): 01-06.

Nirmala, A. (2013): A Study of Workplace Hazards Faced by Workers in Textile Industry as a Part of Occupational Health and Safety. Indian Journal Of Research; 2(4): 333-4. ISSN - 2250-1991.

Pohanish, R. (2012): Sitting's handbook of toxic and hazardous chemicals and carcinogens. $6^{\text {th }}$ ed., U.S.A. William Andrew an imprint of Elsevier; P. 759.

Popov, G., Lyon, B., and Hollcroft, B. (2016): Risk Assessment: A Practical Guide to Assessing Operational Risks .1 ${ }^{\text {st }}$ ed, U.S.A: John Wiley \& Sons; P p 249- 250. ISBN: $1118911040,9781118911044$.

Rantanen, J. (2004): A healthy and safe workplace. African Newsletter on Occupational health and safety; 14 (2): 27.

Rogers, B. (2015): Occupational Health Nursing: Concepts and practice in press. St, Louis: Elsevier, Based Foundations of Nursing in the Community - Elsevier on Vital 
Source: Community-Oriented Practice/edited by: Stanhope M., Lancaster J (2014). $4^{\text {th }}$ ed; P. 945.

Salvendy, G. (2012): Hand Book of Human Factors and Ergonomics. $4^{\text {th }}$ ed., New Jersey, U.S.A: John Wiley \& Sons; P712.ISBN: $1118129067,9781118129067$.

Saramon, S. (2014): A study on exposure related health problems in textile industry.Occp Med Health Aff; 2(3): 67.

Shafik,S., and Abd El-Mohsen, A. (2012): Occupational health: Health Promotion Program to Improve Health Workers in Tourah Cement Factory. Journal of American Science; 8(3): Available at: http://www.americanscience.org.

Sheha, E. (2009): Occupational hazards among workers in Glass manufacture industries. Un Published Master Thesis. Faculty of nursing, Ain Shams University.

Singh, Z. (2015): Health Status of Textile Industry Workers: Prevalence and Socioeconomic Correlates of Different Health Problems. Public Health and Preventive Medicine; 1(3): 137-143.

Smedley, J., Dick F., and Sadhra, S. (2013): Oxford Handbook of occupational Health. $2^{\text {nd }}$ ed, China: Oxford University Press; P p 6- 8. ISBN: 0199651620, 9780199651627.

Stanhope, M. and Lancaster, J. (2016): Public Health Nursing - Elsevier on Vital Source: Population-Centered Health Care in the Community. $9^{\text {th }}$ ed., U.S.A: Elsevier Health Sciences; P948. ISBN: 0323321542, 9780323321549.

Suryakar, A., Katkam, R., Dhadke, V,et al. (2010): A study of oxidative stress in cotton industry workers from Solapur city. Biomedical; 21 (3): 260-264.

Tadesse, S., Kelaye, T., and Assefa, Y. (2016): Utilization of personal protective equipment and associated factors among textile factory workers at Hawassa Town, Southern Ethiopia. Journal of occupational Medicine and Toxicology; 11(6): 1-6.

Takele, G., Haimanot, R., and Martelletti, P. (2008): Prevalence and burden of primary headache in Akaki textile mill workers, Ethiopia. J Headache Pain; 9(2): 119128. DOI 10.1007/s10194-008-0024-Z. 
Zabaniotou, $A$. and Andreou, K. (2010): Development of alternative energy sources for GHG emissions reduction in the textile industry by energy recovery from cotton ginning waste. J. Cleaner Product; 18: 784-790. DOI: 10.1016/j.jclepro.2010.01.006. 


\section{المخاطر المهنية الخاصة بالعمال في مصنع الغزل و النسيج في مدينة دمياط}

\section{عطية مجاهد ابراهيم ، أ.د / عفت محمد القرملاوى، أ.ج.د/ مني عبد الصبور حسن ،}

\section{د/ فاطمة الإمام حافظ}

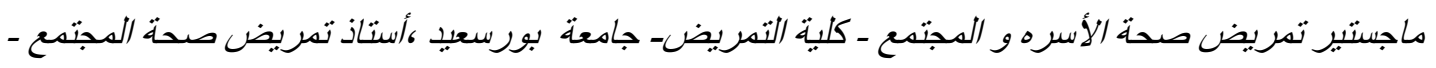

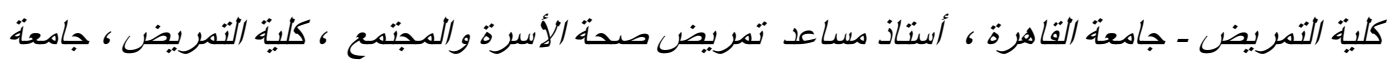
بورسعيد، مدرس تمريض صحة الأسرة والمجتمع - كلبة التمريض- جامعة بورسعبيد

\section{الخـلاصــة}

ان المخاطر المهنية التي توجد داخل مصنع الغزل و النسيج لها تأثير ها الخطير والمدمر علي صحة العمال. أجريت هذة الدراسة بهدف تقييم المخاطر المهنية الموجوده في مصنع الغزل و النسيج في مدينة دمياط و واشتملت مجموعة الدراسه علي 1 • 1 عاملا ، وكان المنهج المستخدم هو المنهج الوصفي وتم جمع البيانات باستخدام أثنين

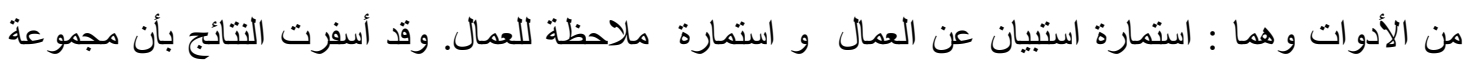

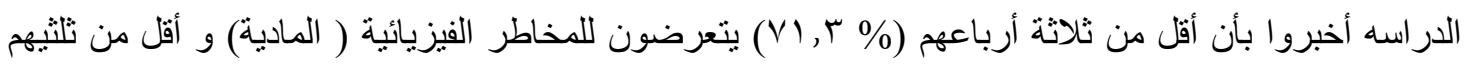

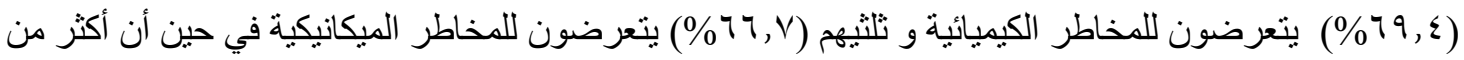

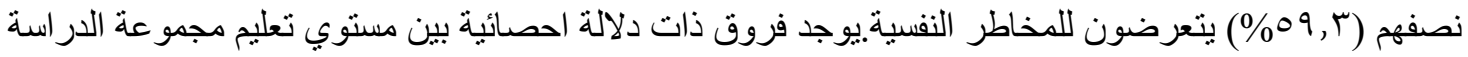

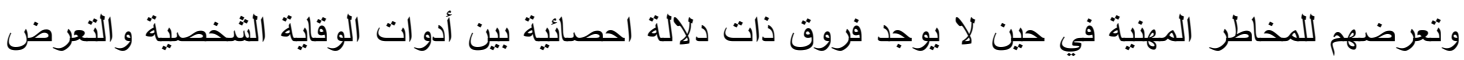
للمخاطر المهنية ــ وقد خلصت الدراسة بأن معظم العمال يتعرضون للمخاطر المهنية المختلفة و التي لها نأثير ها

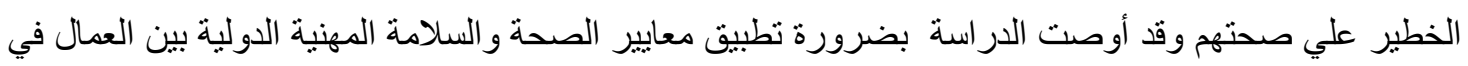
بيئة العمل وذلك لتحسين أداؤهم صحيا ومهنيا.كما أوصت الدراسة أيضا بالتأكيد علي ضرورة ورئ وأهمية استخدام أدوات وأجززة الوقاية بين العمال بالطريقة الصحيحة . الكلـــات السمـشــة : الدخاطر الصحبة الدهنية ، أدوات الوقاية الثخصية ، مصنع الغزل والنسيج 